\title{
Effect of Hydrostatic Pressure and Temperature on Quantum Confinement of AlGaN/GaN HEMTs
}

\author{
Rajab Yahyazadeh $^{1 *}$, Zahra Hashempour ${ }^{1}$ \\ ${ }^{1}$ Department of Physics, Khoy Branch, \\ Islamic Azad University, P.O.Box: 175-58135, IRAN \\ *Corresponding Author
}

DOI: https://doi.org/10.30880/jst.2021.13.01.001

Received 28 November 2020; Accepted 18 March 2021; Available online 18 April 2021

\begin{abstract}
In this paper, an analytical model for quantum confinement electron density in two-dimensional quantum well, has been investigated. In order to obtain the exact AlGaN/GaN HEMTs parameters such as electron density, the wave function, band gap, polarization charge, effective mass and dielectric constant, the hydrostatic pressure and temperature effects are taken into account. It has been found that the electron density decreases with increasing temperature and increases with increasing hydrostatic pressure. With increasing hydrostatic pressure, the effective mass decreases and the quantum confinement electrons are increased in the quantum well. Also with increasing hydrostatic pressure, the height of wave functions increase and decreases electron wave functions to penetrate the quantum barrier but increasing the temperature behaves the opposite of increasing the pressure. However, with increasing temperature, the effective mass is increased and the quantum confinement electrons are reduced. The calculated results for electron density are in good agreement with existing experimental data.
\end{abstract}

Keywords: Hydrostatic pressure, temperature, quantum confinement, effective mass

\section{Introduction}

AlGaN/GaN high electron mobility transistor (HEMT) devices are considered to be very promising candidates for high-speed and high-power applications [1,2]. These devices offer advantages such as high breakdown voltage, high charge density, and good electron mobility [3-5]. The formation of the 2-D electron gas (2DEG) in these devices is the heart of the device operation and has been studied in great detail in the literature. Considering the high degree of application of transistors in electronic components, the effect of hydrostatic pressure and temperature on its performance is important. The external mechanical stress test on these transistors has been carried out by [5]. In the works, the electron density of the quantum well is solved numerically, without considering the electron density of the AlGaN barrier. In order to accurately investigate the effect of hydrostatic pressure and temperature on the possibility of the penetration of the electron wave function into the quantum barrier and quantum confinement, an analytical expression for 2DEG density $n s$ is required. In this expression, the amount of electron density that penetrates the barrier should be determined. The expression should be also physics based, which obviates the use of a large number of empirical parameters. To the best of the authors' knowledge, currently available models for $n s$ are primarily based on numerical calculations, semiempirical model expressions, or simplifying approximations [6-11and 12].

However, they do not consider the effect of the electron charge in the AlGaN barrier layer and hence these models are not valid for the entire range of operation. The electron charge in the AlGaN barrier layer can be neglected only at low and moderate gate biases [11]. In this paper, a unified analytical charge control model for AlGaN/GaN High Electron Mobility Transistor (HEMTs) is considered, which is continuous and valid for all regions of operation is presented. That is capable of determining the effects of hydrostatic pressure and temperature on 2DEG density, electron wave function electron confinement. Also, the dependence of effective mass, dielectric constants, energy gap and conduction band offset 
are considered in terms of pressure and temperature. In the One-dimensional analytical simulations the experimental results, material and device details and all other material parameters have been taken from Refs, 9 and 11 - 13 for $A l_{0.26} G a_{0.74} N / G a N$ HEMTs.

\section{Modeling Hemts}

\subsection{Device Structure}

One-dimensional numerical simulations of the $A l_{x} G a_{1-x} N / G a N$ HEMT were performed using the structure illustrated in Fig. 1. Where the $\mathrm{x}$-direction is along the 2DEG channel, the $\mathrm{z}$-direction is along the growth direction, and the regions I and III represent the ungated channel portions of the HEMT. The structure consists of an undoped GaN layer to form 2DEG channel, an undoped AlGaN spacer layer $\left(d_{s}\right)$, an n-doped AlGaN layer of the thickness $\left(d_{a}\right)$ and undoped Schottky cap layer of the thickness $\left(d_{i}\right)$. In general, the thickness of the AlGaN layer is equal to

$d_{A l G a N}=d_{S}+d_{a}+d_{i}$. It should be notified that to compare with experimental data the structure parameters may vary with the existing experimental data, that is the $\left(d_{s}\right)$ and $\left(d_{i}\right)$ may be neglected. The conduction band diagram on the left side shows the position of the quantum well $(\mathrm{z}=0)$ formation. The gate is a Ni/Pt alloy deposited on a thin $\mathrm{AlGaN}$ layer, of typical thickness $25.5 \mathrm{~nm}$, while the thickness of the underlying GaN layer is about $1 \mu \mathrm{m}$. This causes the AlGaN layer to be completely depleted of mobile charge carriers due to the overlap of the depletion regions at the metal/AlGaN interface (Schottky contact) and the AlGaN/GaN heterostructure interface [13-16]. This ensures that most of the drain current flows through the GaN layer. The source and drain are metal contacts that extend to the GaN layer the gate-source spacing $\left(L_{G S}\right)$ and gate-drain spacing $\left(L_{G D}\right)$ are fixed to $8 \mu \mathrm{m}$. Source and drain lengths $\left(L_{S}, L_{D}\right)$ are taken to be $2 \mu m$ each, and the gate length $\left(L_{G}\right)$ is $4 \mu \mathrm{m}$. It should be noted that miss-match between networks 2 and 3 produces electric fields related to surface polarization between dam and quantum well, the details of which are in Appendix A.

\subsection{Electron Density}

In the analytical model, the temperature and pressure dependence relationships of parameters such as: bound charge at the hetero-interface $\left(\sigma_{b}\right)$ [17,18], lattice constants [19-22], dielectric constant [23,24], AlGaN barrier thickness [25], electron effective mass [26] and band gaps [27] are used. One of the exact analytic relations of density of electron of quantum well is as follows [29]

$$
\begin{gathered}
\mathrm{n}_{\mathrm{S}}=\left[A V_{G O} /(1+B)\right]\left(1-A^{2 / 3} \gamma_{0} /\left[(1+B)^{2 / 3} V_{G o}^{1 / 3}\right]\right) \\
E_{F 2}=q B V_{G O} /(1+B)+A^{2 / 3} \gamma_{0} q V_{G 0}^{2 / 3} /(1+B)^{5 / 3} \\
V_{G 0}=V_{G S}-V_{O F F}-V_{C H}
\end{gathered}
$$

Where $A=\varepsilon_{G a N} / q d_{A l G a N}, B=A / q D, \gamma_{0}\left(=2.12 \times 10^{-12} e V \cdot m^{4 / 3}[30]\right)$ is a parameter that accounts for the assumption of quasi-constant electric field under triangular well approximation and $D$ is the 2-D density of state inside the quantum well at interface (with a typical value of $10^{14} \mathrm{~cm}^{-2} \mathrm{eV}^{-1}$ [ 28 ] ). To calculate the exact electron density in a quantum well, we need to have an electron density in the quantum barrier that has the following relations [29]:

$$
n_{B}=\frac{\varepsilon_{A l G a N}\left(1-A \beta V_{G 0} / \sigma_{s}\right)\left[0.5\left(z-\left(z^{2}+0.25 \delta\right)^{1 / 2}\right)-0.25 \delta\right]}{q c\left[\sigma_{s}-\left[A V_{G 0} /(1+B)\right]\left(1-A^{\frac{2}{3}} \gamma_{0} /\left[(1+B)^{2 / 3} V_{G 0}^{1 / 3}\right]\right)\right]}
$$

where $c=0.27, \quad z=E_{F 2}-\Delta E_{C}+k T \ln (c)$, and $\delta$ is a smoothing parameter. A value of 0.01 for $\delta$ ensures a sufficiently sharp transition of $\mathrm{z}$ between 0 and $\left(z=E_{F 2}-\Delta E_{C}+k T \ln (c)\right)$. The electron density in a quantum well curve 
versus to the source voltage is divided into three regions; 1) $V_{\text {OFF }}<V_{G S}<\mathrm{V}_{\mathrm{Gth}}+\mathrm{V}_{\mathrm{OFF}}$, 2) $\mathrm{V}_{\mathrm{Gth}}+\mathrm{V}_{\mathrm{OFF}}<V_{G S}<V_{G, \text { Barrier }}+V_{O F F}$ and 3) $V_{G, \text { Barrier }}+V_{O F F}>V_{G S}$. The value of $V_{G 0}$ required for device to be at boundary between regions 1 and 2, is denoted by $\mathrm{V}_{\mathrm{Gth}}$ and calculated using from following equation [6]:

$$
V_{G t h}=(D k T \ln 2) \frac{q d_{A l G a N}}{\varepsilon_{G a N}}+\gamma_{0}(D k T)^{2 / 3}(\ln 2)^{2 / 3}
$$

While that required for device to be boundary between regions 2 and 3 is denoted by $V_{G \text {,Barrier }}$ and is calculated by putting $E_{F 2}=\Delta E_{C}$ in Eq. 2 and solving for it. Thus, the required $V_{G S}$ for the device to be at boundaries between regions 1 and 2 , and region 2 and 3 , are given by $\left(\mathrm{V}_{\mathrm{Gth}}+\mathrm{V}_{\mathrm{OFF}}\right)$ and $\left(V_{G, \text { Barrier }}+V_{O F F}\right)$, respectively. By calculating the electron density in a quantum well, a complete analytic relationship called unified Electron Density is as follows [29]:

$$
n_{S, \text { unified }}=\frac{2 k T A \ln \left[1+\exp \left(q V_{G 0} / 2 k T\right)\right]}{q\left[A V_{G 0} /\left(n_{s, \text { Above }-V_{\text {OFF }}}\right)+(A / D) \exp \left(-q V_{G 0} / 2 k T\right)\right]}
$$

The unified model for $\mathrm{n}_{\mathrm{S}}$ valid for $V_{G S}>V_{O F F}$, is denoted by $n_{S, \text { Above-unified }}$, and is given by: $n_{S, \text { Above }-V_{\text {OFF }}}=n_{S}-n_{B}[28]$, where $\mathrm{n}_{\mathrm{S}}$ and $n_{B}$ are given by (1) and (3).

\subsection{Wave Function Penetration Probability}

To calculate the probability of the penetration of the electron wave function into the quantum barrier, we need to use the analytical relationship of the modified Fang-Howard [31]. When the effective mass in quantum well $m_{w}$ and barrier layer $m_{b}$ are not widely different, such as $\mathrm{GaN}-\mathrm{AlGaN}$ hetrostructure, the first order corrections of electron energy in any sub-band quantum well are given by [31]:

$$
\Delta E_{i}=\frac{\hbar^{2} k_{\perp}^{2}}{2}\left[\frac{1}{m_{w}}\left[1-P_{b}\left(E_{i}\right)\right]+\frac{1}{m_{b}} P_{b}\left(E_{i}\right)-\frac{1}{m_{i}}\right]
$$

Where $P_{b}\left(E_{i}\right)$ is the probability of finding 2D electron in the barrier at the level with energy $E_{i}$, and $m_{i}$ corresponding to value of experimentally obtained $m_{e}$. In the case of flat band first order energy shift will be vanish if:

$$
\begin{gathered}
\frac{1}{m_{i}}=\frac{1}{m_{w}}\left[1-P_{b}\left(E_{i}\right)\right]+\frac{1}{m_{b}} P_{b}\left(E_{i}\right) \\
P_{b}\left(\psi_{b}\right)=\int_{-d_{\text {AlGaN }}}^{0} d z\left|\psi_{b}\right|^{2}(8) \\
P_{w}\left(\psi_{w}\right)=\int_{0}^{d_{\text {GaN }}} d z\left|\psi_{w}\right|^{2}
\end{gathered}
$$

The probability calculation of finding $2 \mathrm{D}$ electron in the barrier and quantum well is the following form

$$
\begin{gathered}
P_{b}=\frac{P_{b}\left(\psi_{b}\right)}{P_{b}\left(\psi_{b}\right)+P_{w}\left(\psi_{w}\right)} \\
P_{w}=\frac{P_{w}\left(\psi_{w}\right)}{P_{b}\left(\psi_{b}\right)+P_{w}\left(\psi_{w}\right)}
\end{gathered}
$$


Which $P_{w}\left(\psi_{w}\right)$ and $P_{b}\left(\psi_{b}\right)$ are the quantum well wave function of the in the n-th sub-band the and the wave function penetrating towards the quantum barrier, respectively. That this value of the penetrating wave function is the criterion for calculating the quantum confinement that is effective on the effective masses of the electron.

\section{Results and Discussion}

In this paper, we present an analytical model for calculating the quantum confinement of the AlGaN/GaN field effect transistors, which simultaneously investigates the influence of the hydrostatic pressure and temperature. Fig. 2 shows the temperature dependences on the conduction band offset, the band gaps of AlGaN and GaN heterostructure. Conduction band offset at the interface $A l_{0.26} G a_{0.74} N / G a N$ is inversely proportional to the temperature. The increase in the temperature with a range of $300-600 \mathrm{~K}$ lead to the decrease of the conduction band offset from 0.614 to $0.426 \mathrm{eV}$ in $0 \mathrm{GPa}$. This is attributed to a decrease in the band gap energy of $\mathrm{GaN}$ and $\mathrm{AlGaN}$ with a temperature increase. This phenomenon can be explained by two processes: the modification of the interatomic distances of the crystal lattice by this thermal expansion and the preponderant one linked to the electron-phonon system interaction. This Fig. 2 illustrates that the conduction band offset increases from 0.614 to $1.254 \mathrm{eV}$ in room temperature when the hydrostatic pressure increases in the range of $0-30 \mathrm{GPa}$ which is due to the rising pressure effects on the energy gaps of AlGaN and GaN. The insert in Fig. 2 shows the hydrostatic pressure dependences on the conduction band offset with a range of 0-5GPa. Fig. 3 shows the dependence of the threshold voltage $\left(V_{O F F}\right)$ on the pressure at different temperatures. According to this Fig. 3, the increases in pressure cause an increase of $\left|V_{O F F}\right|$ and increases in temperature causes a decrease in $\left|V_{O F F}\right|$. Due to the dependence of the threshold voltage on the bound charge at the heterointerface $\left(\sigma_{b}\right)$, the variations in the $\sigma_{b}$ versus pressure at different temperatures are shown in the insert in Fig 3. The increase in pressure increases $\sigma_{b}$, this is due to an increase in the polarization of piezoelectric and spontaneous polarization. Fig. 4 and 5 indicate the variation in the electron density of the well $n_{S}$ and the barrier $n_{B}$ versus the gate-source voltage at different pressures. According to Fig. 4 and 5, the increase in pressure causes an increase of $n_{S}$ and a decrease of $n_{B}$. Also, absolute of threshold voltage increases with increasing pressure and with changes in pressure, the regions $V_{O F F}<V_{G S}<\mathrm{V}_{\mathrm{Gth}}+\mathrm{V}_{\mathrm{OFF}}$, $\mathrm{V}_{\mathrm{Gth}}+\mathrm{V}_{\mathrm{OFF}}<V_{G S}<V_{G, \text { Barrier }}+V_{\text {OFF }}$ and $V_{G, \text { Barrier }}+V_{O F F}>V_{G S}$ change that the values of these changes are included in Table 1. According to the values with increasing pressure, the changes in the regions are roughly the same, but these variations are in two opposite directions. An increasing the pressure, decreases the $n_{B}$ (i.e., towards negative gate voltages) and increases the $V_{G, \text { Barrier }}+V_{O F F}$ (toward positive gate voltages). This increase in region $V_{G, \text { Barrier }}+V_{O F F}>V_{G S}$ indicates a decrease in density $n_{B}$ and a decrease in electron penetration with an increase in pressure. Fig. 6 shows the electron wave functions in the quantum well in terms of distance in different gate voltages. In the Fig 6 , with the increase of the gate voltage, the height of wave functions $\left(\psi_{\max }\right)$ and electron density of the quantum well increases. The insert in Fig. 6 shows the performance of the electrons that are impenetrable to the AlGaN barrier. In Fig. 7, the wave functions of the quantum well at different hydrostatic pressure and constant temperatures are shown. In this Fig, with increasing hydrostatic pressure, the height of wave functions and the corresponding electron density increase. To conclude accurately, the numerical values obtained from the graphs are completely written in Table 1. According to Fig. 7 and Table 1, the increase in hydrostatic pressure causes decreases electron wave functions to penetrate the quantum barrier, leading to a decrease in effective mass and a decrease in $n_{S}$. By comparing the Fig. 7 with Fig. 6 , an increase in hydrostatic pressure is similar to the virtual gate of $+0.5 \mathrm{~V}$ of operation. The increase in hydrostatic pressure of $30 \mathrm{GPa}$; reduces the penetration of wave functions from 3800 to 2800 , increase $n_{S}$ to the amount $1.05 \times 10^{12} \mathrm{~cm}^{-2}$ and decrease $n_{B}$ to the amount $0.48 \times 10^{12} \mathrm{~cm}^{-2}$. In Fig. 8, the wave functions of the quantum well at different temperatures and without hydrostatic pressure are shown. In this Fig. 8, with increasing temperature, the height of wave functions and the corresponding electron density decrease. By comparing this Fig. 8 with Fig. 6, an increase in temperature is similar to the virtual gate of $-1 \mathrm{~V}$ of operation. As the Fig. 8 shows, the increase in temperature causes more electron wave functions to penetrate the quantum barrier, leading to an increase in effective mass and a decrease in density. As the temperature rises to $600 \mathrm{~K}$, the penetration of wave functions rises from 3500 to 4800 . Also, $n_{S}$ is reduced to $2.27 \times 10^{12} \mathrm{~cm}^{-2}$ and $n_{B}$ to $0.38 \times 10^{12} \mathrm{~cm}^{-2}$. 


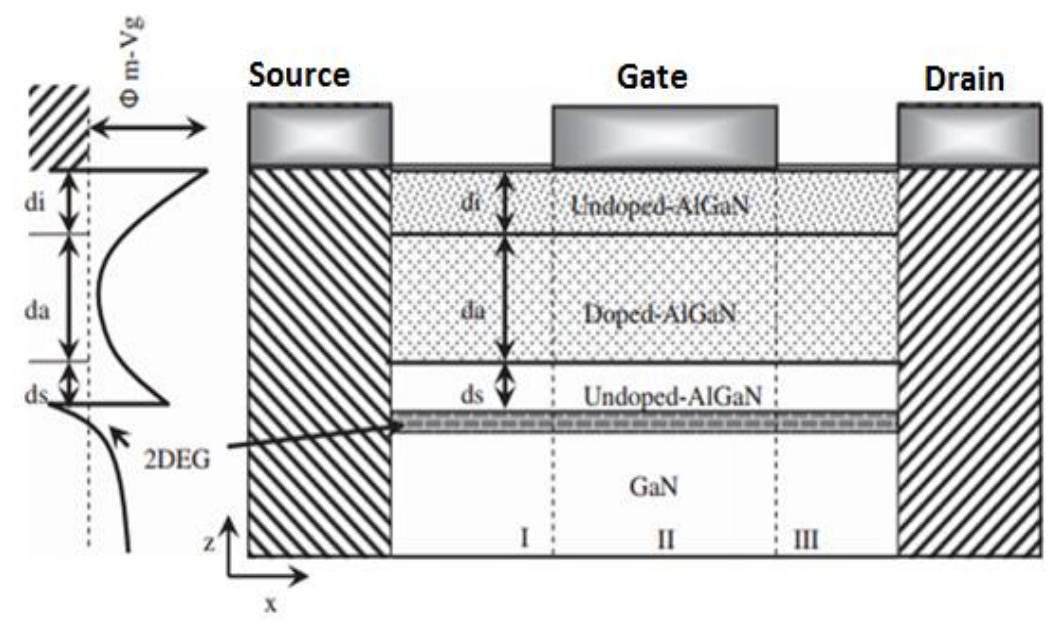

Fig. 1 - Schematic diagram of $A l_{x} G a_{1-x} N$ / GaN HEMT

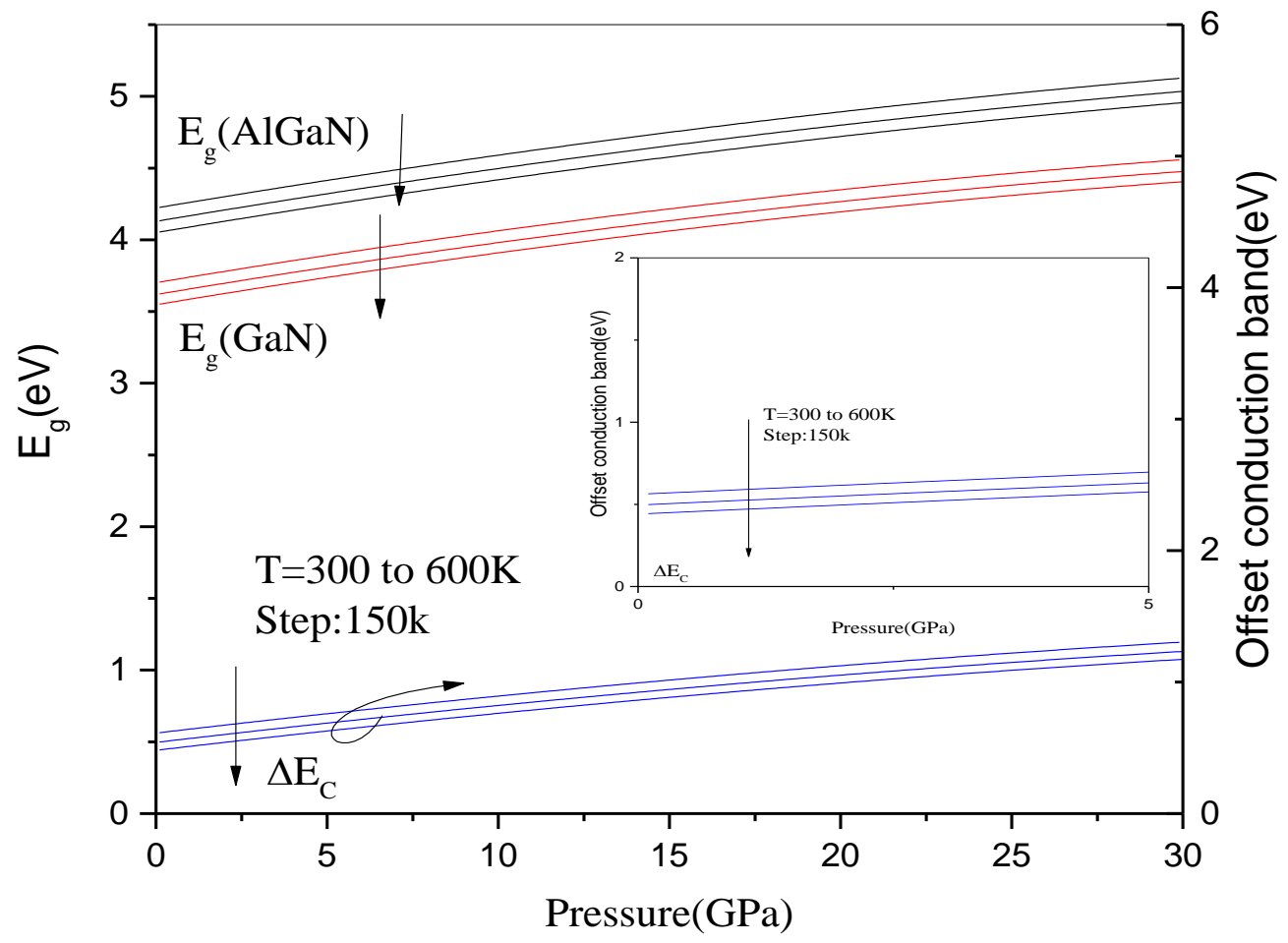

Fig. 2 - The band-gaps energy of $A l_{0.26} G a_{0.24} \mathrm{~N}$, GaN and conduction band offset of the $A l_{0.26} G a N / G a N$ HEMTa as a function of pressure and temperature 


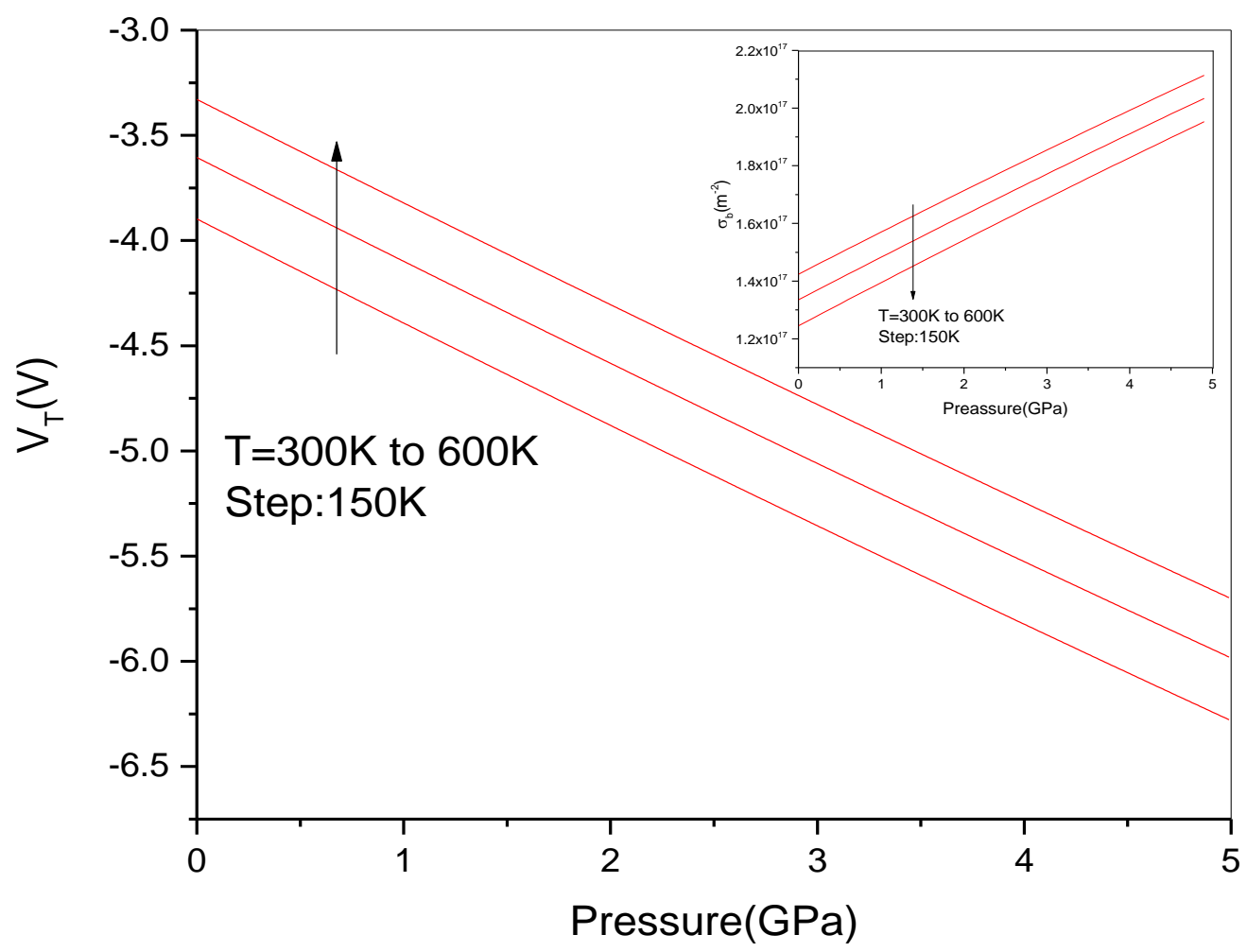

Fig. 3 - The variations of the threshold voltage as a function of the hydrostatic pressure at different temperatures. Insert: variations in bound charge at the hetrointerface $\left(\sigma_{b}\right)$ versus pressure at different temperatures.

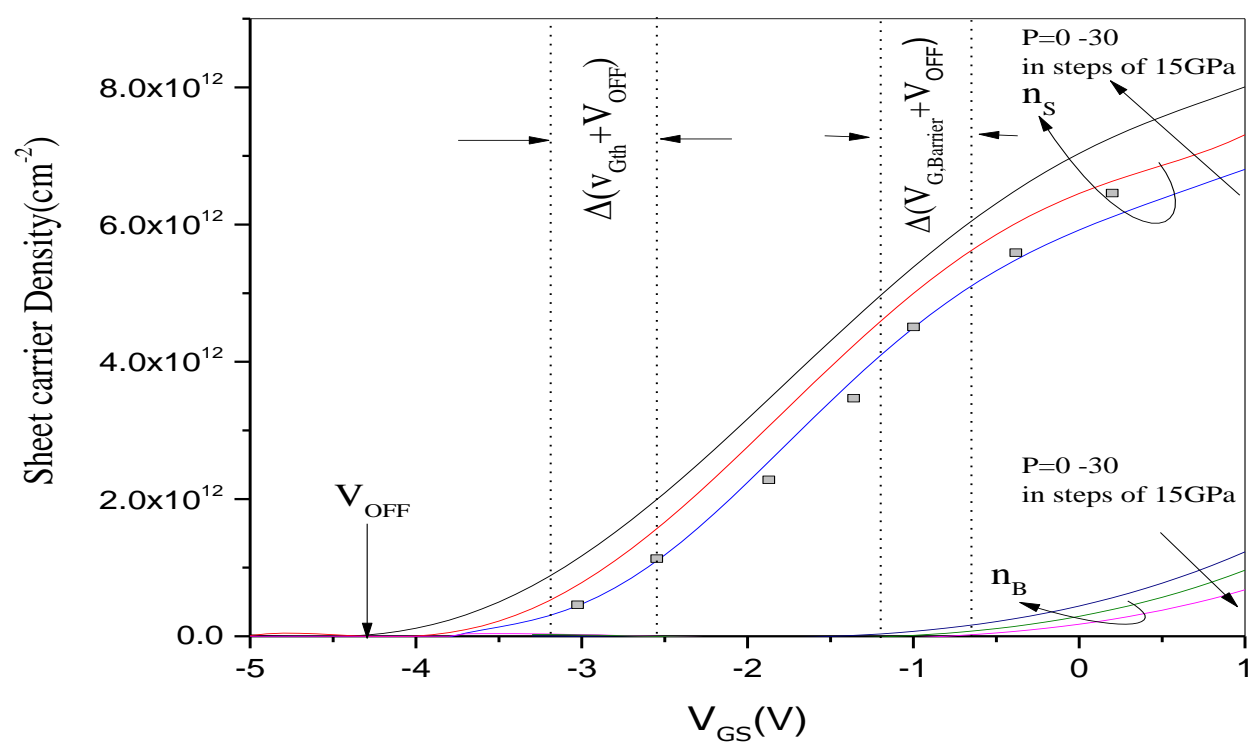

Fig. 4 - The electron density in the well $\left(n_{S}\right)$ and the barrier $n_{B}$ versus the gate-source voltage at different pressures. The experimental data and other needed parameters have been taken from Refs [32]. 


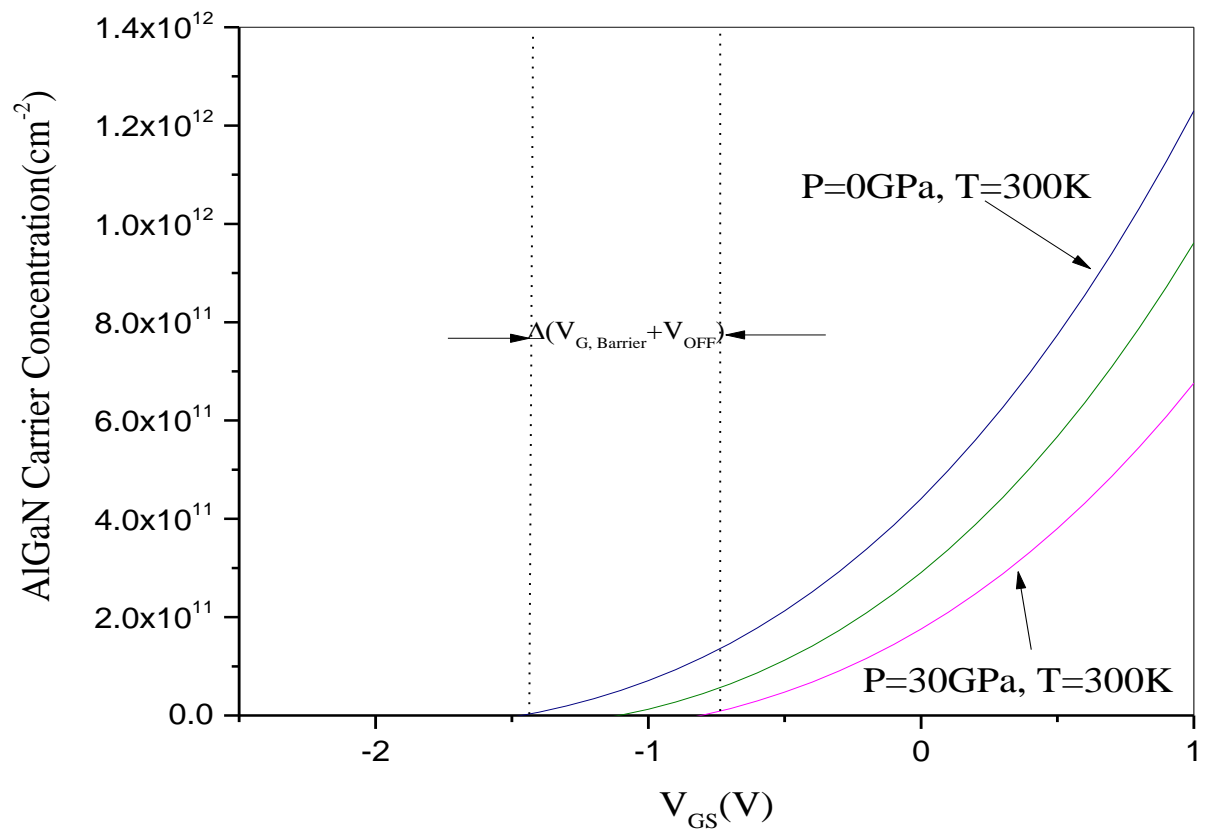

Fig. 5 - The electron density of the barrier $\left(n_{B}\right)$ versus the gate-source voltage at different pressures.

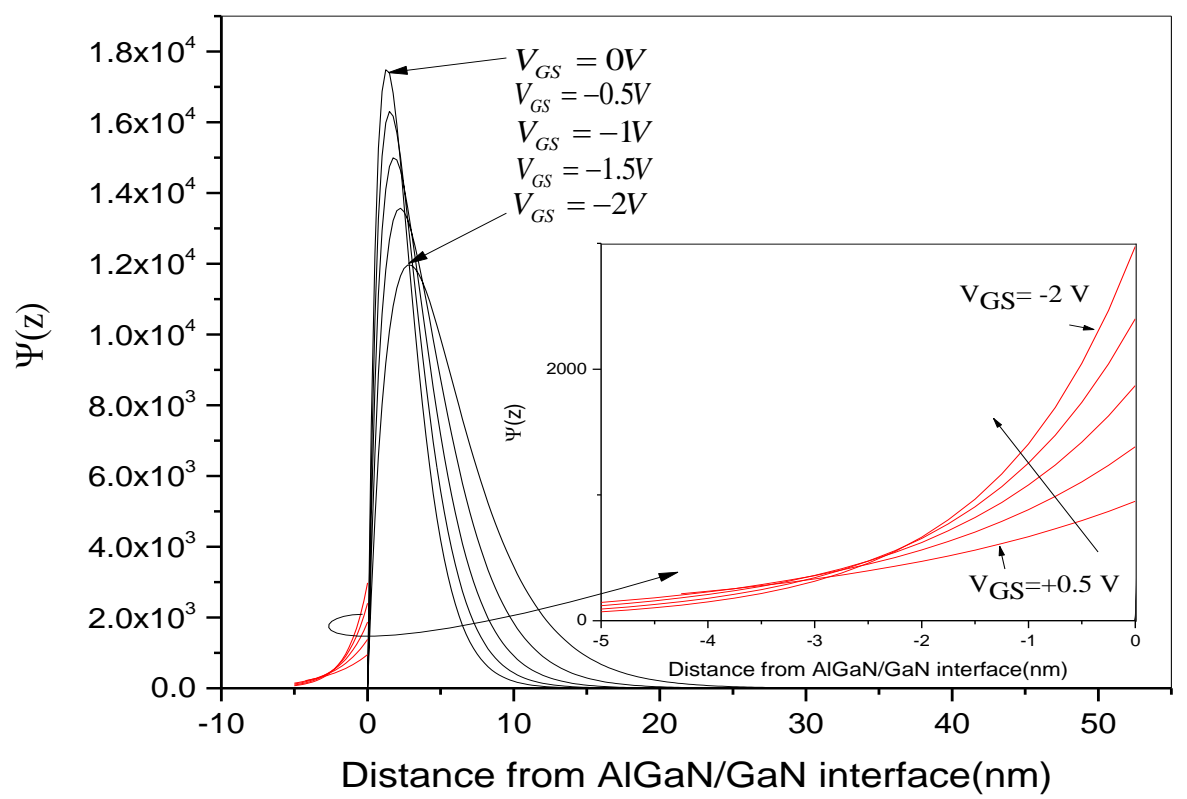

Fig. 6 - The electron wave function $\psi(\mathrm{z})$ as a function of the distance from AlGaN/GaN interface under different $n_{S \text {,unified }}$ (here $n_{S \text {,unified }}$ corresponds to the electron density under the gate region as a function of gate-source bias). The insert indicates the electron wave function impenetrate to the AlGaN barrier. 


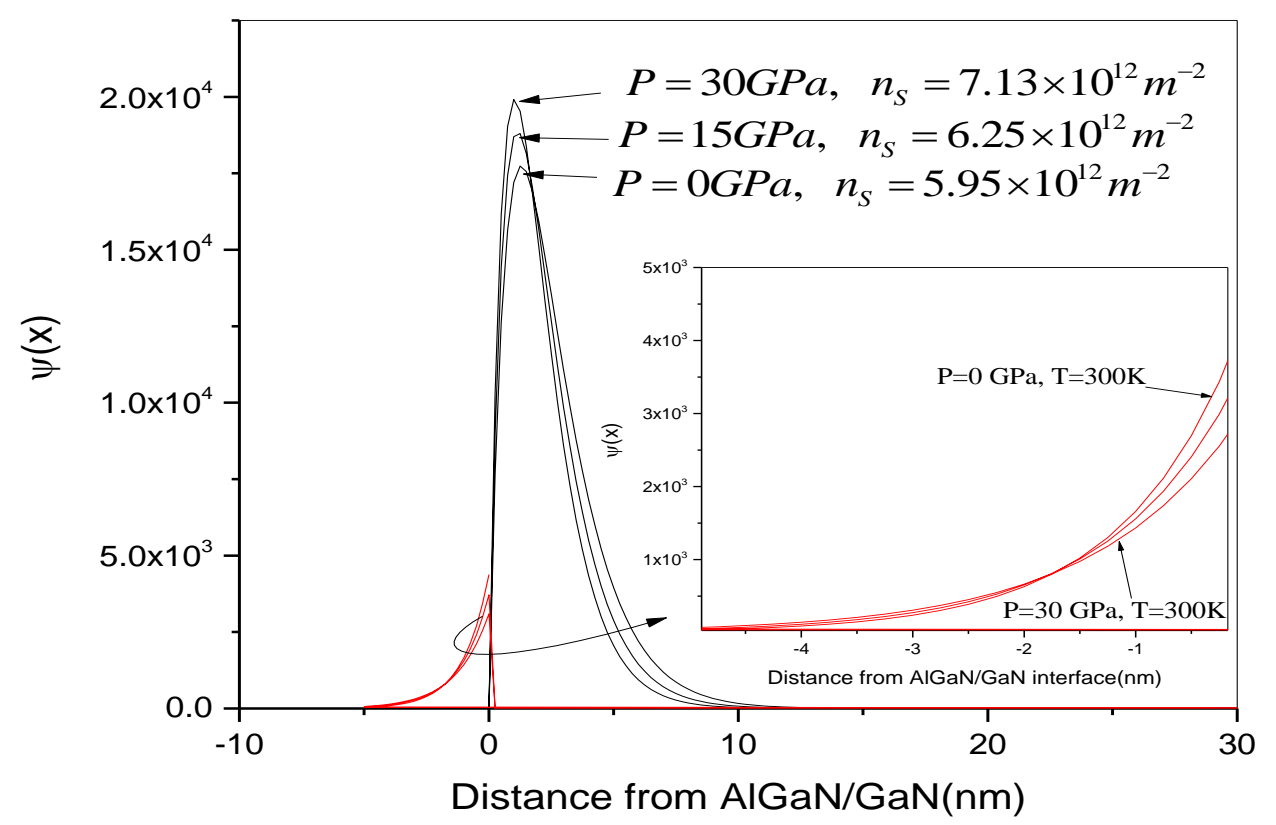

Fig. 7 - The electron waves function $\psi(z)$ as a function of the distance from AIGaN/GaN interface under different hydrostatic pressure and $T=300 \mathrm{~K}$. The insert indicates the electron wave function impenetrate to the AlGaN barrier.

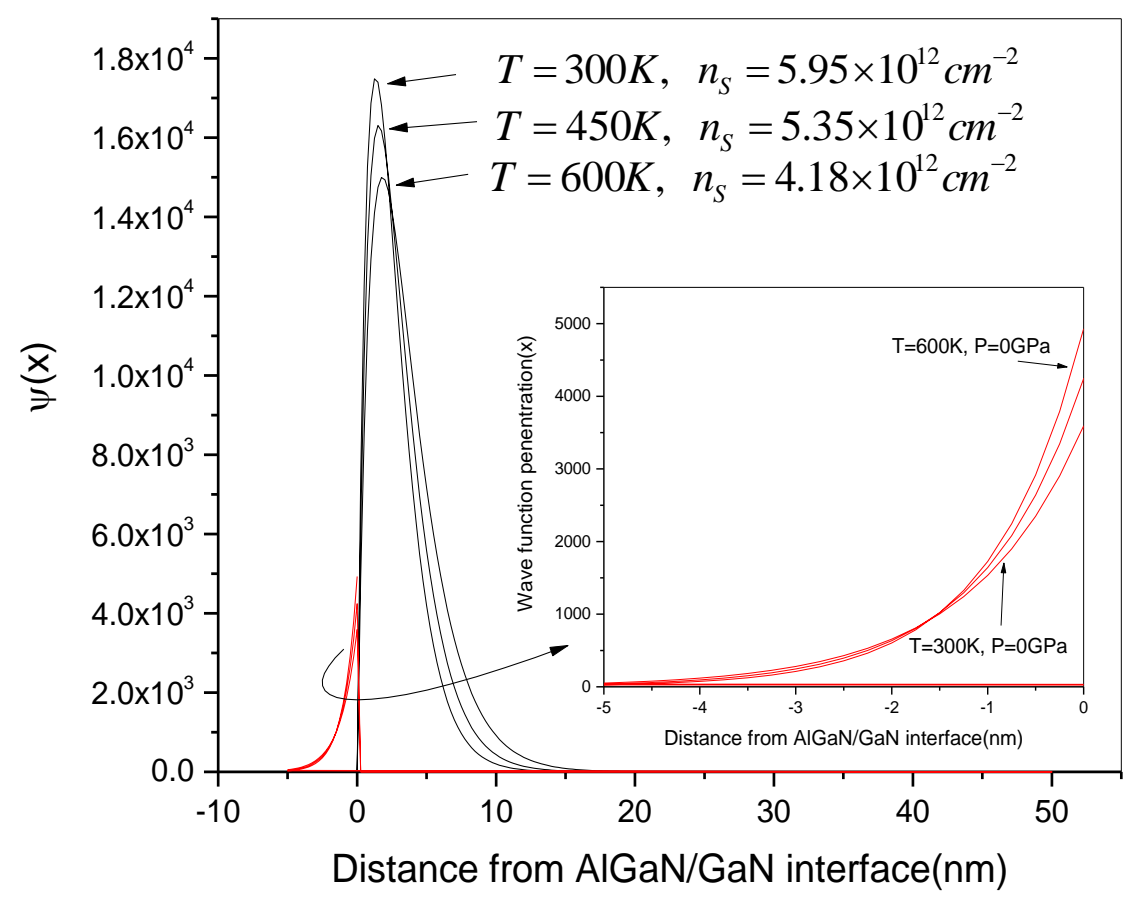

Fig. 8 - The electron waves function $\psi(z)$ as a function of the distance from AlGaN/GaN interface under different temperature and without hydrostatic pressure. The insert indicates the electron wave function impenetrate to the AlGaN barrier. 
Table 1 - Calculated parameters of 2DEG in AIGaN/GaN HEMTs in different temperature.

\begin{tabular}{cccccccc}
\hline $\mathbf{T}(\mathbf{K})$ & $n_{S}\left(10^{12} \mathrm{~cm}^{-2}\right)$ & $n_{B}\left(10^{12} \mathrm{~cm}^{-2}\right)$ & $\psi_{\max }$ & $P_{b}(\%)$ & $m_{w}^{*}\left(m_{0}\right)$ & $\mathrm{V}_{\mathrm{Gth}}+\mathrm{V}_{\mathrm{OFF}}$ & $V_{G, \text { Barrier }}+V_{\text {OFF }}$ \\
\hline $\mathbf{3 0 0}$ & 5.95 & 0.43 & $1.78 \times 10^{4}$ & 7.183 & 0.231 & -2.6 & -1.45 \\
$\mathbf{4 5 0}$ & 5.35 & 0.61 & $1.62 \times 10^{4}$ & 8.872 & 0.233 & -2.4 & -1.65 \\
$\mathbf{6 0 0}$ & 4.18 & 0.79 & $1.51 \times 10^{4}$ & 10.747 & 0.237 & -2.2 & -1.95 \\
\hline
\end{tabular}

Table 2 - Calculated parameters of 2DEG in AIGaN/GaN HEMTs in different hydrostatic pressure.

\begin{tabular}{cccccccc}
\hline $\mathbf{P}(\mathbf{G P a})$ & $n_{s}\left(10^{12} \mathrm{~cm}^{-2}\right)$ & $n_{B}\left(10^{12} \mathrm{~cm}^{-2}\right)$ & $\psi_{\max }$ & $P_{b}(\%)$ & $m_{w}^{*}\left(m_{0}\right)$ & $\mathrm{V}_{\mathrm{Gth}}+\mathrm{V}_{\mathrm{OFF}}$ & $V_{G, \text { Barrier }}+V_{\text {OFF }}$ \\
\hline $\mathbf{0}$ & 5.95 & 0.43 & $1.78 \times 10^{4}$ & 7.183 & 0.231 & -2.6 & -1.45 \\
$\mathbf{1 5}$ & 6.24 & 0.29 & $1.85 \times 10^{4}$ & 5.933 & 0.226 & -2.9 & -1.15 \\
$\mathbf{3 0}$ & 6.65 & 0.125 & $2 \times 10^{4}$ & 3.747 & 0.220 & -3.2 & -0.72 \\
\hline
\end{tabular}

\section{Conclusions}

In this paper, an analytical model for quantum confinement electron density in two-dimensional quantum well of $\mathrm{AlGaN} / \mathrm{GaN}$ HEMTs has been investigated. From the results, as the temperature rises to $600 \mathrm{~K}$, the penetration of wave functions rises from 3500 to 4800 . Also, $n_{S}$ is reduced to $2.27 \times 10^{12} \mathrm{~cm}^{-2}$ and $n_{B}$ to $0.38 \times 10^{12} \mathrm{~cm}^{-2}$. This increase in temperature is similar to the virtual gate of $-1 \mathrm{~V}$ and the increase in hydrostatic pressure is similar to the virtual gate of $+0.5 \mathrm{~V}$. The increase in pressure of $30 \mathrm{GPa}$; reduces the penetration of wave functions from 3800 to, increase $n_{S}$ to the amount $1.05 \times 10^{12} \mathrm{~cm}^{-2}$ and decrease $n_{B}$ to the amount $0.48 \times 10^{12} \mathrm{~cm}^{-2}$. Also, the amount of $\left|V_{O F F}\right|$; increases with increasing pressure. Increasing pressure, the changes in the three regions are roughly the same, but these variations are in two opposite directions. An increasing pressure decreases the $n_{B}$ (i.e., towards negative gate voltages) and increases the $V_{G, \text { Barrier }}+V_{O F F}$ (toward positive gate voltages).

\section{Acknowledgement}

We are a faculty member of Islamic Azad University, Khoy Branch, and all resources are provided to our work through the University. The authors would like to thank Khoy Branch.

\section{References}

[1] R. Chu, GaN power switches on the rise. (2020). Demonstrated benefits and unrealized potentials, Appl. Phys. Lett., 116, 090502

[2] R. Yahyazadeh, Zahra hashempour. (2019). Numerical Optimization for AlGaN/GaN HEMTs Including polarization coulomb field Scattering, Journal of Non -Oxide Glasses, 11(2), 19-26

[3] R Yahyazadeh, Z. Hashempour. (2019). Numerical Optimization for Source-Drain Channel Resistance of AlGaN/GaN HEMTS, Journal of Science and technology, 11(1), 1-9

[4] R Yahyazadeh. (2018). Analytical- numerical model for sheet resistivity of AlGaN/GaN HEMTs, Journal of Non -Oxide Glasses, 10(2), $57-63$

[5] C. Jiang et al. (2017). Piezotronic effect tuned AlGaN/GaN high electron mobility transistor, Nanotechnology, 28, 455203

[6] G. Hu, L. Lib, Y. Zhang. (2019). Two-dimensional electron gas in piezotronic devices, Nano Energy, 59, 667-673

[7] R. Yahyazadeh, Zahra hashempour. (2020). Numerical Performance of AlGaN/GaN High Electron Mobility Transistors under Hydrostatic Pressure and Temperature. Journal of Science and technology, 12(1), 15-28

[8] I. Angelov, H. Zirath, N. Rorsman, (1992). A new empirical nonlinear model for HEMT and MESFET devices, IEEE Trans. Microw. Theory Tech, 40(12), 2258-2266 
[9] Z.H. Zhang, J.H. Yuan, K.X. Guo. (2018). The Combined Influence of Hydrostatic Pressure and Temperature on Nonlinear Optical Properties of $\mathrm{Ga}_{0.7} \mathrm{Al}_{0.3} \mathrm{~N} / \mathrm{GaAs}$ Morse Quantum Well in the Presence of an Applied Magnetic Field, Materials, 11, 668

[10] M. Li, Y. Wang. (2008). 2-D analytical model for current-voltage characteristics and transconductance of AlGaN/GaN MODFETs, IEEE Trans. Electron Devices, 55(1) 261-267

[11] X. Cheng, M. Li, Y. Wang. (2009). Physics-Based Compact Model for AlGaN/GaN MODFETs with Close-Formed I-V and C-V Characteristics, IEEE Trans. Electron Devices, 56(12), 2881-2887

[12] Z. Hashempour, A. Asgari, S. Nikipar, M. Abolhasani, M. Kalafi. (2009). Numerical performance evaluation of $\mathrm{AlGaN} / \mathrm{GaN}$ high electron mobility transistors including gate length effects, Physica E, 41,1517-1521

[13] Y. F. Wu et al. (1996). Bias dependent microwave performance of AlGaN/GaN MODFET's up to $100 \mathrm{~V}$, IEEE Electron Device Lett, 18(6), 290-292

[14] Y. F. Wu et al. (1998) High Al-content AlGaN/GaN MODFETs for ultrahigh performance, IEEE Electron Device Lett, 19(2), 50-53

[15] J. W. Lee, K. J. Webb. (2004). A temperature-dependent nonlinear analytic model for AlGaN-GaN HEMTs on SiC, IEEE Trans. Microw. Theory Techn, 52(1), 2-9

[16] S. Khandelwal et al. (2013). Robust surface-potential-based compact model for GaN HEMT IC design, IEEE Trans. Electron Devices, 60(10), 3216-3222

[17] O. Ambacher, A. B Foutz, J Smart, J. R Shealy, N. G Weimann, K Chu, et al. (2000). Two dimensional electron gases induced by spontaneous and piezoelectric polarization in undoped and doped AlGaN/GaN heterostructures, J. Appl. Phys, 87,334

[18] O. Ambacher, J. Majewski, C. Miskys, et al. (2002). Pyroelectric properties of Al (In) GaN/GaN hetero- and quantum well structures, J. Phys. Condens. Matter, 14, 3399

[19] Z J Feng, Z J Cheng, H Yue. (2004). Chinese Physics, 13, 1334

[20] V. Fiorentini, F. Bernardini, and O. Ambacher. (2002) Evidence for nonlinear macroscopic polarization in III-V nitride alloy Heterostructures, Appl. Phys. Lett, 80, 1204

[21] P. Perlin, L. Mattos, N. A. Shapiro, J. Kruger, W. S. Wong, T. Sands, N. W. Cheung, and E. R. Weber. (1999) Reduction of the energy gap pressure coefficient of GaN due to the constraining presence of the sapphire substrate, J. Appl. Phys, 85, 2385

[22] K. elibol, G. Atmaca, P. Tasli, S.B.Lisesivdin. (2013). A numerical study on subband of InxAl1-xN/InN-based HEMT structure with low-indum $(x<0.01)$ barrier layer, Solid state communication, 162, 8-12

[23] C. M. Duque, A. L. Morales, M. E. Mora-Ramos, and C. A. Duque. (2015). Exciton-related optical properties in zinc-blende GaN/InGaN quantum wells under hydrostatic pressure. Physica Status Solidi (b), 252, 670

[24] M. Yang et al. (2016). Effect of polarization coulomb field scattering on parasitic source access resistance and extrinsic transconductance in AlGaN/GaN heterostructure FETs. IEEE Trans. Electron Devices, 63, 1471-1477

[25] K.J Bala, A. J Peter, and C. W Lee. (2017). Simultaneous effects of pressure and temperature on the optical transition energies in a $G a_{0.3} I n_{0.7} N / G a N$ quantum ring, Chemical Physics, 495 (2017) 42

[26] I. Vurgaftman, J. R Meyer, L. R. R Mohan. (2001). Band parameters for III-V compound semiconductors and their alloys, J. Appl. Phys, 89, 5815

[27] N. E. Christensen, I. Gorczyca. (1994). Optical and structural properties of III-V nitrides under pressure, Phys. Rev. B, 504397

[28] N. Karumuri, S. Turuvekere, N. DasGupta, A. DasGupta. (2014). A Continuous Analytical Model for 2-DEG Charge Density in AlGaN/GaN HEMTs Valid for All Bias Voltages, IEEE Trans. Electron Devices, 61(7), 23432349.

[29] N. S. Swamy, A. K. Dutta. (2018). Analytical models for the 2DEG density, AlGaN layer carrier density, and drain current for AlGaN/GaN HEMTs, IEEE Trans. Electron Devices, 65(3), 936

[30] H. K. Kwon et al. (1999). Radiative recombination of two-dimensional electrons in a modulation-doped Al0.37Ga0.63N/GaN single heterostructure, Appl. Phys. Lett, 75(18), 2788-2790

[31] A M Kurakin, S A Vitusevich et al.(2009). Quantum confinement effect on the effective mass in two-dimensional electron gas of AlGaN/GaN heterostructures, J. Appl. Phys, 105, 073703

[32] P.Cui et al. (2018). Effect of different gate lengths on polarization coulomb field scattering potential in AlGaN/GaN heterostructure field-effect transistors, Science reports, 89036 


\section{Appendix A: Electric Fields}

Assuming no free charge, electric displacements at interface between $(j)$ th and $(j+1)$ th layers and between $(j+1)$ th and $(j+2)$ th layers can be expressed as follows:

$$
\begin{gathered}
\varepsilon_{j+1} \varepsilon_{0} F_{j+1}=\varepsilon_{j} \varepsilon_{0} F_{j}+P_{j}-P_{j+1} \\
\varepsilon_{j+2} \varepsilon_{0} F_{j+2}=\varepsilon_{j+1} \varepsilon_{0} F_{j+1}+P_{j+1}-P_{j+2}
\end{gathered}
$$

Substituting from Eq.(A1) gives an expression for Eq.(B2):

$$
\varepsilon_{j+2} \varepsilon_{0} F_{j+2}=\varepsilon_{j} \varepsilon_{0} F_{j}+P_{j}-P_{j+2}
$$

Where, $\varepsilon_{j}$ represents the dielectric constants and $P_{j}$ is the polarization in jth layer. It follows that the field in any layer is related to field in a particular layer as in the following:

$$
F_{k}=\frac{1}{\varepsilon_{k} \varepsilon_{0}}\left(\varepsilon_{j} \varepsilon_{0} F_{j}+P_{j}-P_{k}\right) \quad k=j+2
$$

We focus on the case where there is no voltage difference across MQW either because thermodynamic equilibrium prevails or an applied field exists that compensates any built-in field. The condition, in which the volt value dropped across MQW is zero, is as follows:

$$
\sum_{k} L_{k} F_{k}=L_{j} F_{j}+\sum_{k \neq j} L_{k} F_{k}
$$

Here, $L_{k}$ and $L_{j}$ are the kth and jth layers thickness. Substituting from Eq.(A4) gives an expression for electric field in any layer:

$$
F_{j}=\frac{\sum_{k \neq j}\left(P_{k}-P_{j}\right)\left(L_{k} / \varepsilon_{k}\right)}{\varepsilon_{k} \varepsilon_{0} \sum_{k}\left(L_{k} / \varepsilon_{k}\right)}
$$

In the case of a MQW with only two types of layers ( $L_{w}$ and $L_{b}$ ), the fields are:

$$
F_{w}=\frac{\left(P_{b}-P_{w}\right) L_{b}}{\varepsilon_{0}\left(\varepsilon_{w} L_{w}+\varepsilon_{b} L_{b}\right)} \quad, F_{b}=\frac{\left(P_{w}-P_{b}\right) L_{w}}{\varepsilon_{0}\left(\varepsilon_{w} L_{w}+\varepsilon_{b} L_{b}\right)}=-F_{w} \frac{L_{w}}{L_{b}}
$$

In these equations, the difference in polarization density is equal to surface polarization density in $\mathrm{AlGaN} / \mathrm{GaN}$, which is calculated by the following equation:

$$
\sigma_{b}=\left|P_{b}-P_{w}\right|=\left|P_{A l_{m} G a_{(1-m) N} P Z}^{P Z}+P_{A l_{m} G a_{(1-m)} N}^{S P}-P_{G a N} S P-P_{G a N}^{P Z}\right|
$$

Where,

$$
\begin{gathered}
P_{\text {GaN }}^{P Z}=-0.918 \in+9.541 \epsilon^{2} \quad(\mathrm{~A} 9) \\
P_{G a N}^{P Z}=\left\{\begin{array}{l}
-1.808 \in+5.624 \epsilon^{(2)} \text { for } \in<0 \\
-1.808 \in-7.888 \epsilon^{2} \text { for } \in>0
\end{array} \quad(\mathrm{~A} 10)\right. \\
P_{\left(A l_{m} G a_{(1-m)} \mathrm{N}\right)}^{S P}=0.090 m-0.034(1-m)+0.21 x(1-m)
\end{gathered}
$$

Basal strain is expressed from lattice of substrate $a_{s}$ and epilayer $a_{e}(T, P, m)$ :

$$
\grave{o}(T, P, m)=\frac{a_{c}-a_{e}(T, P, m)}{a_{e}(T, P, m)}
$$

Here, $a_{e}(T, P, m)$ represents the lattice constants 